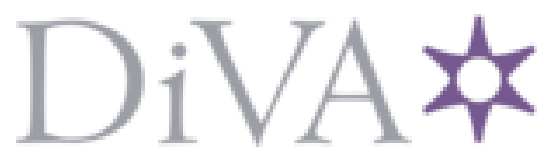

http://www.diva-portal.org

This is the published version of a paper published in International Journal of Sports Medicine.

Citation for the original published paper (version of record):

Díaz, M., Bocanegra, O L., Teixeira, R R., Espindola, F S. (2012)

Response of salivary markers of autonomic activity to elite competition

International Journal of Sports Medicine, 33(9): 763-768

https://doi.org/10.1055/s-0032-1304638

Access to the published version may require subscription.

N.B. When citing this work, cite the original published paper.

Permanent link to this version:

http://urn.kb.se/resolve?urn=urn:nbn:se:rkh:diva-2796 


\section{Response of Salivary Markers of Autonomic Activity to Elite Competition}

Authors

Affiliations
M. M. Diaz , O. L. Bocanegra ${ }^{1}$, R. R. Teixeira', S. S. Soares ${ }^{2}$, F. S. Espindola

${ }^{1}$ Institute of Genetics and Biochemistry, Federal University of Uberlandia, Brazil

${ }^{2}$ Faculty of Physical Education, Federal University of Uberlandia, Brazil

\section{Key words \\ saliva \\ exercise \\ stress \\ biomarker}

accepted after revision January 31, 2012

Bibliography DOI http://dx.doi.org/ 10.1055/s-0032-1304638 Published online:

May 11, 2012

Int J Sports Med 2012; 33: 763-768 @ Georg Thieme Verlag KG Stuttgart · New York ISSN 0172-4622

\section{Correspondence}

Dr. Foued Salmen Espindola Instituto de Genética e Bioquímica

Universidade Federal de Uberlândia

Av. Pará 1720

38400982 Uberlândia

Brasil

Tel.: +55/34/3218 2477

Fax: +55/34/3218 2203

fouedespindola@gmail.com

\section{Abstract}

$\nabla$

We investigated the response of salivary total protein (TP), alpha-amylase (SAA) and chromogranin A ( $\mathrm{CgA})$ to sporting competition and their relation with positive and negative affect. 11 professional swimmers were examined during the first day of a national contest and on a recreated event that matched time-of-the-day and day-of-the-week assessments 2 weeks later. Total protein was determined by the Bradford method and sAA and CgA by Western blotting upon awakening, 30 and 60 min post awakening, immediately before warming up for competition and 5, 20 and $60 \mathrm{~min}$ after competition.

\section{Introduction}

\section{$\nabla$}

The stress response is mediated by components in the autonomic nervous system (ANS) and the hypothalamic-pituitary adreno-cortical (HPA) axis. The ANS stimulates the adrenal medulla to produce catecholamines, whereas glucocorticoids are the final effectors of the HPA axis [41]. More than a decade ago, salivary alpha-amylase (sAA) was suggested as a surrogate marker for autonomic activity given that its release into saliva is elicited by the stimulation of the salivary glands by sympathetic and parasympathetic nerves [33]. Furthermore, research has demonstrated that beta-adrenergic agonists are capable of stimulating SAA release without increasing salivary flow [14] and that beta-blocking agents inhibit sAA secretion in response to psychological challenges [37]. This pattern of evidence has resulted in the incorporation of sAA into a broad series of behavioral studies in which the SAA response to adverse environments has been measured and even successfully applied in clinical [42] and military settings [11].
Psychometric instruments included the Positive Affect and Negative Affect Schedule-X. The concentrations of TP, sAA and CgA differed from controls only prior to and 5 min after the event. We observed positive correlations between higher negative affect scores with higher levels of TP, SAA and CgA prior to the event on the competition day. All 3 markers showed a similar reactivity to sporting competition, which may be attributed to the mechanisms responsible for protein secretion into saliva when collection is performed with no exogenous stimulation. TP is an attractive marker in sports psychology since its determination is faster and cheaper than traditional kinetic or immune assays.
Chromogranin A ( $\operatorname{CgA})$, on the other hand, is costored and co-released with catecholamines from secretory vesicles in the adrenal medulla and post-ganglionic sympathetic axons [45]. Chromogranin A is also produced by the submandibular gland and is secreted into the saliva under autonomic control. In addition, chromogranin A has antifungal and antimicrobial properties $[24,35,40]$. Salivary CgA has received some attention as a marker of psychological stress because similar responses to adverse psychological stimuli, such as SAA, have been reported for salivary CgA. In particular, higher concentrations of $\mathrm{CgA}$ were present in young professors after they gave lectures to university graduates [16], after moderate exercise [2] and following cognitive assessments [21].

Although sAA and CgA are secreted into the saliva from different glands, the parotid and submandibular glands, respectively, the secretion of both proteins into the saliva is under autonomic control. In addition to the parotid and submandibular glands, the sublingual and numerous minor glands also contribute to the secretion and the composition of whole mouth saliva. Essen- 
tially, parasympathetic input to the salivary glands produces most of the fluid in saliva, whereas sympathetic stimulation results in protein secretion [31]. Although this view is somewhat simplistic and exceptions to such patterns of saliva secretion exist, one could expect to observe similar responses of both sAA and $\mathrm{CgA}$ to adverse environments. Similarly, it could be speculated that not only SAA and $\mathrm{CgA}$, but also salivary total protein (TP) would show a similar response to hostile stimuli. From a methodological perspective, measuring TP is faster and cheaper than traditional kinetic and immune-enzymatic assays. Surprisingly, few studies to date have investigated TP as a marker of autonomic activity; most research on salivary biomarkers has focused on sAA.

Professional sports competition offers a unique scenario in which to assess the response to stress. This type of competition involves social comparison and evaluation and is a significant source of pressure for athletes [18]. Secondly, professional sports competitions are highly organized, and the criteria for performance are clear. Therefore, the collection of the data and the assessment of the subjects are objective and standardized. Moreover, because the subjects are assessed in real-life environments, authentic responses to adverse stimuli are observed. It is well established that competition induces sympathetic arousal both in the anticipation of and in the response to the event $[3,23]$. Such variation in autonomic activity is related to the perceived nature of the competition and the demands posed by the competition. Thus, the subjects may experience feelings ranging from worry and fear to vigor and aggressiveness depending on how they cope with stress. To a large extent, a lower reactivity of the HPA axis and the ANS system has been associated with positive affect [13], whereas a higher reactivity is related to negative affect $[1,33]$.

In this study, we examined the variation in salivary TP, sAA and $\mathrm{CgA}$ in professional swimmers during a national contest and on a regular training day. In theory, a higher autonomic drive would be present on the day of the competition compared with the non-competition day due to higher components of anxiety and pressure. Thus, we hypothesized that 1) higher concentrations of TP, sAA and CgA would be observed on the competition day relative to the control day and that these higher concentrations would be associated with higher scores in mood disturbance, and 2) no difference amongst the levels of TP, sAA and CgA would be present on the competition day or on the control day.

\section{Methods}

$\nabla$

\section{Subjects}

The subjects were 11 male professional swimmers (aged 21.5 \pm 2.16 years; BMI: $22.7 \pm 2.5 \mathrm{VO}_{2}$ max: $52.7 \pm 3.2 \mathrm{ml} / \mathrm{kg} . \mathrm{min}$; competition experience: $8.7 \pm 2.8$ years). The subjects were recruited from a team before a national swimming competition. The performance of each athlete in these competitions is used to partially define his status on the team and his salary. None of the subjects smoked or was taking any kind of medication during the study. Two weeks before the competition, the subjects were informed of the experimental procedures and provided their written informed consent. The experimental protocol was in compliance with the Ethical Standards in Sports and Exercise Science Research [19] and was approved by the Institutional Review Board.

\section{Experimental design}

The subjects were asked to collect saliva samples throughout the first day of a 1-week national competition. A total of 7 saliva samples were collected at the following times: (T1) upon awakening (while still lying in bed), (T2) $30 \mathrm{~min}$ later, (T3) $1 \mathrm{~h}$ later [approximately $0800 \mathrm{~h}$ ], (T4) immediately before warming up for competition [approximately $1600 \mathrm{~h}$ ], (T5) 5, (T6) 20 and (T7) $40 \mathrm{~min}$ after the competition [approximately $1900 \mathrm{~h}$ ]. Morning samples (T1-T3) were included to explore the probable relations between the awakening response of salivary proteins and mood disturbance induced by competition.

The subjects were instructed to refrain from eating, drinking and tooth brushing during the first hour after awakening, or at least $1 \mathrm{~h}$ before T4. They were also asked to refrain from drinking alcohol or caffeinated beverages for at least $24 \mathrm{~h}$ prior to the days of saliva collection. The subjects were given clear and concise directions regarding collection procedures and the importance of punctuality. The subjects were asked to record collection times for the first 3 samples, and those subjects who provided samples deviating more than $10 \mathrm{~min}$ from the appropriate times were excluded from the analyses. The remaining 4 samples were collected under the supervision of 2 researchers at the precise times. 2 weeks after the competition, the event was recreated at the swimming team's training facilities to obtain control values. The subjects performed on the same day of the week and at the same time of the day as that of the real competition. Performance (swimming time) was recorded and compared between the actual competition and the control competition. The subjects were encouraged by their coaches to perform with the same intensity and at similar swimming times during the control day. The water temperature $\left(25-28^{\circ} \mathrm{C}\right)$ was also controlled to match that of the competition.

\section{Measures}

\section{Saliva sampling and handling}

Whole mouth saliva was collected into collection vials with no exogenous stimulation. The saliva was allowed to pool in the mouth and then drooled into pre-weighted conical polypropylene tubes after $2 \mathrm{~min}$. Immediately after the event (before T5), the subjects were given $70 \mathrm{~mL}$ of distilled water to wash their mouths. The subjects were asked to spit the water and to swallow to empty the mouth before saliva was collected. The samples were placed on ice, transported to the laboratory and stored frozen at $-20^{\circ} \mathrm{C}$ until the analysis was performed.

\section{Western blotting for salivary alpha-amylase and chromogranin A}

On the day of analysis, the samples were thawed and centrifuged at $3000 \mathrm{rpm}$ for $15 \mathrm{~min}$. The concentration of the total protein in the samples was used as loading control. Total protein was determined using the Bradford method. It involves the binding of proteins to Coomassie brilliant blue, to form a protein-dye complex that shifts the absorption maximum of the dye from 465 to $595 \mathrm{~nm}$. The increase in absorption is directly proportional to the amount of protein in the sample. Absorption read at $595 \mathrm{~nm}$ is recorded [8]. All of the samples from each subject were assayed on the same plate in duplicate. To avoid the possible effects of salivary flow rate on the concentration of proteins, especially after exercising (dehydration), 10 micrograms of the total protein from each sample were denatured under reducing conditions and applied on 5-20\% SDS-polyacrylamide gradient 


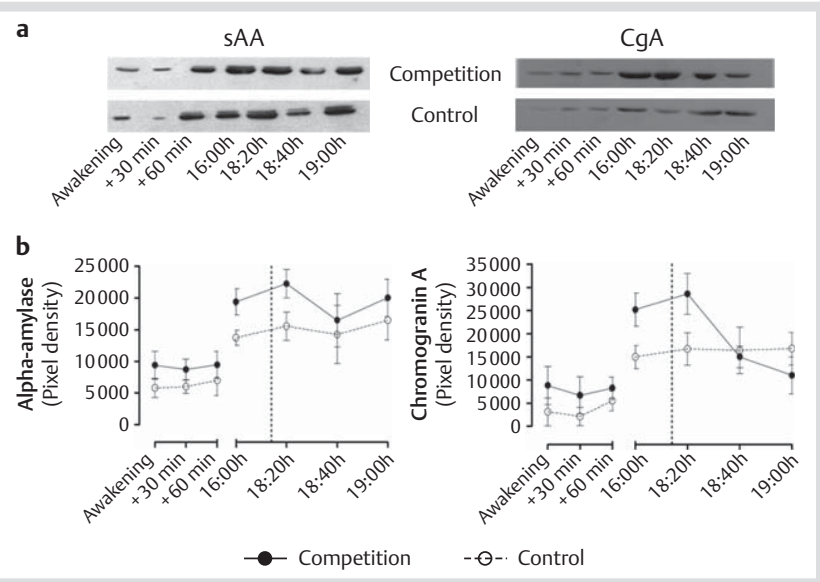

Fig. 1 Diurnal profile of sAA and $\mathrm{CgA}$ during competition and control in swimmers. a shows representative results from blots of sAA and CgA. b summarizes the quantitative results of the variation in the concentration of sAA and $\mathrm{CgA}$. The dotted vertical line indicates time of swimming. Values are means and error bars indicate SD.

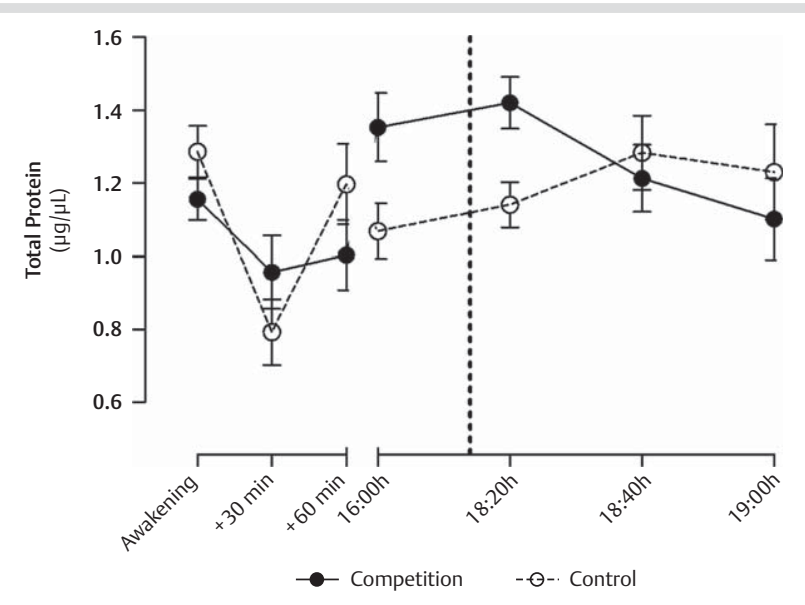

Fig. 2 Diurnal profile of TP during days of competition and control in swimmers. The dotted vertical line indicates time of swimming. Values are means and error bars indicate SD.

gels, as previously suggested [28]. The proteins were separated and then transferred onto nitrocellulose membranes in transfer buffer (25 mM Tris, $190 \mathrm{mM}$ glycine, $20 \% \mathrm{MeOH}, \mathrm{pH}$ 7.8-8.4) for $2 \mathrm{~h}$ at $200 \mathrm{~mA}$ and $4{ }^{\circ} \mathrm{C}$. The protein transfer was confirmed by visualization with Ponceau. The membranes were blocked for $4 \mathrm{~h}$ at $4^{\circ} \mathrm{C}$ in blocking buffer (5\% non-fat dry milk in PBS w/v). The membranes were then incubated overnight at $4{ }^{\circ} \mathrm{C}$ with purified polyclonal rabbit anti-human SAA (dilution 1:5000) (produced in our laboratory) and mouse monoclonal antihuman CgA (dilution 1:1000) (Millipore, Temecula, CA), respectively, and subsequently incubated with secondary antibodies for $2 \mathrm{~h}$. After the incubations with specific primary and then secondary antibodies, the labeled proteins were detected with ECL reagents and by exposing the developed blots to GE Healthcare films. Densitometrical analysis of the spots was performed using ImageJ (U. S. National Institutes of Health, Bethesda, Maryland, USA) by a researcher who was blinded to the experimental groups (competition vs. control). The area in pixels of each spot was determined in triplicate, and the means were used for statistical analyses.

\section{Psychometric instruments}

The subjects completed the Positive and Negative Affect Schedule-X (PANAS-X), immediately after collecting saliva at times T2, $\mathrm{T} 4$ and T6. The PANAS-X is a self-report measure of positive and negative affect composed of 11 separate 5-point Likert scales in which the subjects rate the extent to which they are currently experiencing each emotion [44]. Cronbach's alpha value was 0.84 and 0.86 for the Positive and Negative Affect scales, respectively. The PANAS-X had been previously translated into Portuguese and the results are highly correlated to the original instrument [30].

\section{Statistical analyses}

The data were tested for normality using the Shapiro-Wilk test prior to the analyses. No transformations were necessary for any of the variables. The concentration of TP, SAA and CgA at each sampling time was averaged and compared between the competition and the control day using paired $t$ tests. Psychometric scores and performance (swimming times) were also compared using paired $t$ tests. The area under the curve of the diurnal profile of TP, sAA and CgA was calculated using the trapezoid formula [32]. Spearman's rank correlation coefficient was used to compare the psychological and the physiological variables. For all of the analyses, the significance level was $p<0.05$. The results shown are the means (SD) unless otherwise stated.

\section{Results}

$\boldsymbol{\nabla}$

\section{Averaged concentrations of salivary markers of sympathetic activity}

- Fig. 1, 2 show the diurnal profile of TP, sAA and CgA during the competition day and the control day. In general, all of the markers displayed a similar pattern with a distinct decrease $30 \mathrm{~min}$ post-awakening with following increasing concentrations. We did not observe significant differences in the concentrations of $\mathrm{TP}$, sAA and CgA in the morning period (T1-T3) or at the end of the day (T6-T7). Differential changes, however, were observed at times T4 [TP: $t(14)=2.67, \mathrm{p}=0.0182$; $\mathrm{sAA}: t(9)=5.44, \mathrm{p}=0.0004$; CgA: $t(6)=3.42, p=0.014$ ] as well as at T5 [TP: $t(14)=4.42$, $\mathrm{p}=0.0006 ;$ sAA: $t(11)=3.53, \mathrm{p}=0.0048 ; \operatorname{CgA}: t(10)=4.30$, $\mathrm{p}=0.0015]$ when compared to the control times.

\section{Diurnal profile of salivary markers of sympathetic activity during competition}

Here we set out to investigate whether the diurnal course of TP, sAA and CgA was different between the day of the competition and the control day. Areas under the curve with respect to ground (AUCg) [TP: $t(4)=0.95, p=0.39$; $s A A: t(6)=1.11, p=0.31$; CgA: $t(4)=0.48, p=0.65]$ and with respect to increase (AUCi) [TP: $t(8)=1.4, \mathrm{p}=0.17 ; \mathrm{sAA}: t(4)=4.2, \mathrm{p}=0.07 ; \operatorname{CgA}: t(4)=1.53$, $\mathrm{p}=0.19$ ] did not differ from the control group for any variable.

\section{Affect scores and performance}

- Table 1 shows the positive and the negative affect scores throughout the competition and the control day. Overall, we found different and higher negative scores in the morning and prior to the competition whereas we found different but lower positive scores prior to and after the competition than on the control day. No difference in the swimming times was observed between the competition and the control day [Competition: 53.12 (2.02) s; Control: 53.43 (1.88) s; $t(6)=0.64, \mathrm{p}=0.76]$. 
Table 1 Positive and negative affect scores during competition and control. Values in the upper line (bold) indicate scores during competition whereas values in the lower line indicate scores during control. Values are means (SD).

\begin{tabular}{|llll|}
\hline Collection Times & T2 & T4 & T6 \\
\hline negative affect & $\mathbf{1 8 . 6 ( 3 . 6 )}$ & $\mathbf{2 1 . 6 ( 6 . 9 ) *}$ & $\mathbf{2 0 . 0 ( 7 . 1 )}$ \\
& $10.8(1.0)$ & $12.0(1.7)$ & $15.6(4.5)$ \\
\hline positive affect & $\mathbf{2 8 . 5 ( 5 . 6 )}$ & $\mathbf{2 9 . 8 ( 4 . 8 )}$ & $\mathbf{2 3 . 1 ( 3 . 2 )}$ \\
& $31.0(9.1)$ & $38.0(6.1)$ & $30.7(3.1)$ \\
\hline
\end{tabular}

\section{Correlations between affect and salivary markers}

Here, the averaged concentrations of salivary markers at the times T2, T4 and T6 were correlated with the scores of negative and positive affect. Significant positive correlations were found only at T4 for negative affect on the competition day [TP: $r(11)=0.67, \mathrm{p}<0.05 ; \mathrm{sAA}: r(11)=0.59, \mathrm{p}<0.05: \operatorname{CgA}: r(11)=0.61$, $\mathrm{p}<0.05]$.

\section{Discussion}

$\nabla$

To understand the dynamics of salivary markers of autonomic activity prior to and after competition, the mechanisms of protein secretion into saliva must be considered. In essence, saliva is constantly secreted at a basal rate from 3 major pairs of glands (parotid, submandibular and sublingual) and from numerous minor glands spread over the oral mucosa. However, copious salivary secretion is induced upon sensory and mechanical stimuli by autonomic control to the salivary glands [25]. Whereas parasympathetic nerves are mainly responsible for the secretion of water and electrolytes, sympathetic nerves are predominantly responsible for the secretion of proteins. Exceptions to this include, amongst others, the secretion of sAA from glands that are mainly stimulated by parasympathetic nerves, such as the palate and the sublingual glands [7], and increased salivary secretion of immunoglobulin $A$ as a result of stimuli from both the sympathetic and the parasympathetic nerves to the plasma cells [31]. Nevertheless, evidence suggests that the rate of protein secretion into the saliva by sympathetic stimuli is superimposed upon parasympathetic stimulation when the glands are simultaneously innervated [31]. In addition to this, it is thought that the neural pathways that innervate salivary glands are under the control of the higher centers of the brain, such as the cerebral cortex and the limbic center. Consequently, the composition and volume of saliva is responsive not only to sensory and mechanical stimuli, but also to emotional states such as stress [25].

Consistent with our original hypothesis, we found a marked elevation of TP, sAA and CgA prior to and after the competition. However, in contrast to our expectations, we did not observe differential changes in the concentration of salivary proteins upon awakening and $60 \mathrm{~min}$ later even though higher negative affect scores were reported. Given the extensive experience of the subjects in the competition, they may have engaged mentally in the competition only later in the day as the contest was approaching (T4). Secondly, the magnitude of the feelings experienced early in the morning, although different from the control day, might not have been sufficiently intense to evoke significant sympathetic arousal.

A considerable body of research has been dedicated to assess the response of markers of autonomic activity both before and after adverse environments. Our findings are consistent with those of some studies $[6,10,21,26]$ but not others [22] that have reported significant increases in the activity of SAA and the concentration of $\operatorname{CgA}$ before academic and social evaluations and parachute jumping. The subjects in our study were assessed during a national swimming contest. All of the subjects were professional athletes, and their performance in the competition had significant consequences in regards to their salary and competitive status within their team. Thus, it seems that in situations in which the outcome of the challenge poses serious threats to the self, as seen in the contexts of assessment of job performance or physical danger, a rise in the activity and the concentration of these proteins before taking part in stressful situations could be expected.

It may be argued that in our study, such a rise in TP, sAA and $\operatorname{CgA}$ would be due to a higher sympathetic drive associated with exercise. Nonetheless, the subjects performed within almost identical swimming times on the control day, and it is unlikely that small differences in intensity resulted in such divergent profiles in the concentration of proteins prior to and immediately after the contest. Furthermore, we also found higher negative and lower positive affect scores relative to the control day before the contest. Thus, the disparity in the concentrations of proteins between the days of competition and control was in fact due to the psychological factors associated with the threat to the self posed by the competition.

When a sporting competition is chosen to assess biobehavioral responses, special attention must be paid to salivary flow after exercise because dehydration can strongly influence the concentration of salivary proteins. Lower rates of salivary flow have only been reported after prolonged exercise $(<30 \mathrm{~min})$ [43]. In our study, the subjects were assessed in contests that took no more than $1 \mathrm{~min}$. Nonetheless, as previously suggested [4,28], we addressed this issue by determining the concentration of both sAA and $\mathrm{CgA}$ using the same quantity of protein from each sample $(10 \mu \mathrm{g})$ independently from the volume of saliva collected. Thus, when our results were controlled for protein concentration, our results are in line with recent research that shows peak levels of sAA approximately 5-10 min after adverse stimuli and a subsequent decrease of sAA reaching baseline levels $20-30$ min later $[2,15,22]$.

It is worth repeating at this point that our goal was to investigate the response of salivary proteins in response to adverse psychological stimuli. Since our study was designed so the subjects would serve as their own control, it was necessary that the days of competition and control included the same physical strain [see Results - Performance]. Comparing the diurnal rhythm of salivary proteins on competition days against regular days (no competition or training) or against physically inactive subjects would not have allowed us to determine the effect of psychological adverse stimuli on elite athletes. Especially considering that exercise alone could result in a higher concentration of proteins. By examining the diurnal rhythm of proteins on resting days (in addition to competition and training), we would only be able to define the extent to which exercise leads to a higher secretion of proteins. Furthermore, diurnal rhythms of salivary proteins under physiological situations have been already explored and our findings on lower levels of protein in the morning with increasing concentration during the day corroborate those of the literature $[12,20]$.

Several of the findings of our study are novel. First, this is the first demonstration of the reactivity of TP and $\operatorname{CgA}$ to a profes- 
sional competition. Interestingly, both TP and CgA displayed a similar response to the competition than did sAA with differential changes in anticipation to and after the contest. This may have important repercussions in biobehavioral research and sports psychology. Since the proposition of sAA as a surrogate marker of sympathetic activity, a significant series of studies has assessed the variation in its activity under different conditions. However, from a laboratorial standpoint, determining the concentration of TP is faster, cheaper and more practical than traditional kinetic or immune assays. In this respect, TP has been successfully applied to monitor exercise intensity and hydration status $[4,5,43]$. However, no study thus far has considered TP as a marker of autonomic activity under psychological adversity. As mentioned before, if the rationale behind the use of sAA in biobehavioral research is that SAA is released into saliva upon sympathetic stimulation, higher concentrations of TP are also to be expected after the same challenges.

Secondly, it appears that the levels of TP, sAA and CgA are not strongly associated with variations in negative and positive affect. Most likely, other types of scales with the predominant components of tension, anxiety and excitement as observed in sympathetic arousal would have been more appropriate to detect the same magnitude of variation between affect and salivary proteins.

Finally, distinct, challenging psychological stimuli represented here by a professional competition only seem to override the regular rhythm of salivary proteins prior to and immediately after the contest. In accordance with the morning profile of our data, there are previous studies that have reported diurnal rhythms of sAA and CgA with nadir concentrations early in the morning and an awakening response with a steep decrease 30 min after awakening $[12,38]$.

On the other hand, the results of this study have to be interpreted in light of some limitations. In general, the stress-induced changes in TP, sAA and CgA are congruent with the autonomic arousal previously reported during competition $[3,23]$. However, the reader is cautioned not to consider salivary proteins as exclusive read-outs of sympathetic activity. Parasympathetic innervation to the salivary glands regulates flow rate and as already noted, might also result in protein secretion. Although stress-induced protein release into saliva appears to be independent of flow rate [34], we are not able to differentiate additive effects, if any, between the 2 branches of the ANS. Secondly, we used a sample of subjects of modest size. We chose to assess the response to stress in a professional sporting competition because it poses significant psychological and physiological demands on the subjects. Additionally, it elicits genuine responses to stress because subjects are assessed in real-life situations. It is usually difficult to include a larger sample size in experimental studies because there are few professional teams with larger and more homogenous groups of athletes. In addition, experimental designs often interfere with training sessions or competition events. To compensate for sample size, we designed a comprehensive protocol that allowed us to observe timely variations in the concentrations of protein prior to and in response to the task. Furthermore, the subjects were all male, within a narrow age range and with similar levels of performance and extensive experience in competition. Additionally, baseline values were obtained from the same subjects in a carefully recreated event that matched the time-of-the-day and the day-of-the-week assessments. Several other studies on the variation in salivary constituents in response to stress and exercise have included similar if not smaller samples $[9,15,17,26,27,29$, $36,39]$ and some have reported equivalent results to our study. Thus, although it would be desirable to work with a larger group, we believe, based on previous research, that our experimental design and the characteristics of the subjects that little, if any, difference in the dynamics of TP, SAA and CgA would have been observed with a larger population.

\section{Conclusions \\ $\nabla$}

Taken together, these data indicate that TP, sAA and CgA show a similar pattern of reactivity towards professional competitions. If our supposition is correct and proteins, irrespective of their function or their mechanisms of secretion, are released into saliva mostly upon sympathetic drive, TP could represent a very attractive marker of autonomic activity in biobehavioral research given the simplicity of the Bradford assay. Changes in the concentration of TP, sAA and CgA become apparent only moments before and after taking part in a competition and do not strictly reflect alterations in negative and positive affect.

\section{Acknowledgements}

$\nabla$

This study was supported by grants from the funding agency FAPEMIG. M.D. and O.B. received graduate fellowships from CNPq and the international program PEC-PG.

We are grateful to participants for their involvement and understanding towards the instructions given throughout the study. Thanks are also due to Mr. G. Degani and Mr. W. Pires for their support and consideration to include members of their team to conduct this work.

\section{References}

1 Adam EK, Hawkley LC, Kudielka BM, Cacioppo JT. Day-to-day dynamics of experience - cortisol associations in a population-based sample of older adults. Proc Natl Acad Sci USA 2006; 103: 17058-17063

2 Allgrove JE, Gomes E, Hough J, Gleeson M. Effects of exercise intensity on salivary antimicrobial proteins and markers of stress in active men. J Sports Sci 2008; 26: 653-661

3 Baron R, Petschnig R, Bachl N, Raberger G, Smekal G, Kastner P. Catecholamine excretion and heart rate as factors of psychophysical stress in table tennis. Int J Sports Med 1992; 13: 501-505

4 Bocanegra OL, Diaz MM, Teixeira RR, Soares SS, Espindola FS. Determination of the lactate threshold by means of salivary biomarkers: chromogranin A as novel marker of exercise intensity. Eur J Appl Physiol (January 7, 2012) doi:10.1007/s00421-011-2294-4

5 Bortolini MJS, De Agostini GG, Reis IT, RPMS Lamounier, Blumberg JB, Espindola FS. Total protein of whole saliva as a biomarker of anaerobic threshold. Res Q Exerc Sport 2009; 80: 604-610

6 Bosch JA, Brand HS, Ligtenberg TJ, Bermond B, Hoogstraten J, Nieuw Amerongen AV. Psychological stress as a determinant of protein levels and salivary-induced aggregation of Streptococcus gordonii in human whole saliva. Psychosom Med 1996; 58: 374-382

7 Bosch JA, Veerman ECI, de Geus EJ, Proctor GB. $\alpha$-Amylase as a reliable and convenient measure of sympathetic activity: don't start salivating just yet! Psychoneuroendocrinology 2011; 36: 449-453

8 Bradford MM. A rapid and sensitive method for the quantitation of microgram quantities of protein utilizing the principle of protein-dye binding. Anal Biochem 1976; 72: 248-254

9 Bullock N, Cox AJ, Martin DT, Marino FE. Resting salivary and plasma cortisol in elite athletes following long-haul travel from Australia to Canada. J Sci Med Sport 2009; 12: 300-302

10 Chatterton RTJr, Vogelsong KM, Lu YC, Hudgens GA. Hormonal responses to psychological stress in men preparing for skydiving. J Clin Endocrinol Metab 1997; 82: 2503-2509 
11 Cosenzo KA, Fatkin LT, Patton DJ. Ready or not: enhancing operational effectiveness through use of readiness measures. Aviat Space Environ Med 2007; 78: B96-B106

12 Den $R$, Toda $M$, Ohira $M$, Morimoto $K$. Levels of awakening salivary $\mathrm{CgA}$ in response to stress in healthy subjects. Environ Health Prev Med 2011; 16: 155-157

13 Dockray S, Steptoe A. Positive affect and psychobiological processes. Neurosci Biobehav Rev 2010; 35: 69-75

14 Ehlert U, Erni K, Hebisch G, Nater U. Salivary alpha-amylase levels after yohimbine challenge in healthy men. J Clin Endocrinol Metab 2006; 91: 5130-5133

15 Filaire E, Alix D, Ferrand C, Verger M. Psychophysiological stress in tennis players during the first single match of a tournament. Psychoneuroendocrinology 2009; 34: 150-157

16 Filaire E, Dreux B, Massart A, Nourrit B, Rama LM, Teixeira A. Salivary alpha-amylase, cortisol and chromogranin $A$ responses to a lecture: impact of sex. Eur J Appl Physiol 2009; 106: 71-77

17 Fortes $M B$, Whitham M. Salivary Hsp72 does not track exercise stress and caffeine-stimulated plasma Hsp72 responses in humans. Cell Stress Chaperones 2011; 16: 345-352

18 Gucciardi DF, Dimmock JA. Choking under pressure in sensorimotor skills: Conscious processing or depleted attentional resources? Psychol Sport Exerc 2008; 9: 45-59

19 Harriss DJ, Atkinson G. Update - ethical standards in sport and exercise science research. Int J Sports Med 2011; 32: 819-821

20 Hong RH, Yang YJ, Kim SY, Lee WY, Hong YP. Determination of appropriate sampling time for job stress assessment: the salivary chromogranin A and cortisol in adult females. J Prev Med Public Health 2009; 42: 231-236

21 Kanamaru Y, Kikukawa A, Shimamura K. Salivary chromogranin-A as a marker of psychological stress during a cognitive test battery in humans. Stress 2006; 9: 127-131

22 Kivlighan KT, Granger DA. Salivary alpha-amylase response to competition: relation to gender, previous experience, and attitudes. Psychoneuroendocrinology 2006; 31: 703-714

23 Kraemer WJ, Fry AC, Rubin MR, Triplett-McBride T, Gordon SE, Koziris LP, Lynch JM, Volek JS, Meuffels DE, Newton RU, Fleck SJ. Physiological and performance responses to tournament wrestling. Med Sci Sports Exerc 2001; 33: 1367-1378

24 Lugardon K, Raffner R, Goumon Y, Corti A, Delmas A, Bulet P, Aunis D, Metz-Boutigue MH. Antibacterial and antifungal activities of vasostatin-1, the N-terminal fragment of chromogranin A. J Biol Chem 2000; 275: $10745-10753$

25 Mese H, Matsuo R. Salivary secretion, taste and hyposalivation. J Oral Rehabil 2007; 34: 711-723

26 Nater UM, Rohleder N, Gaab J, Berger S, Jud A, Kirschbaum C, Ehlert U. Human salivary alpha-amylase reactivity in a psychosocial stress paradigm. Int J Psychophysiol 2005; 55: 333-342

27 Noto Y, Kudo M, Hirota K. Back massage therapy promotes psychological relaxation and an increase in salivary chromogranin A release. J Anesth 2010; 24: 955-958

28 de Oliveira VN, Bessa A, Lamounier RPMS, de Santana MG, de Mello MT, Espindola FS. Changes in the salivary biomarkers induced by an effort test. Int J Sports Med 2010; 31: 377-381

29 Oliver SJ, Laing SJ, Wilson S, Bilzon JLJ, Walsh NP. Saliva indices track hypohydration during $48 \mathrm{~h}$ of fluid restriction or combined fluid and energy restriction. Arch Oral Biol 2008; 53: 975-980
30 Peluso MAM, Savalli C, Cúri M, Gorenstein C, Andrade LH. Mood changes in the course of preparation for the Brazilian university admission exam - a longitudinal study. Revista Brasileira de Psiquiatria 2010; 32: $30-36$

31 Proctor GB, Carpenter GH. Regulation of salivary gland function by autonomic nerves. Auton Neurosci 2007; 133: 3-18

32 Pruessner JC, Kirschbaum C, Meinlschmid G, Hellhammer DH. Two formulas for computation of the area under the curve represent measures of total hormone concentration versus time-dependent change. Psychoneuroendocrinology 2003; 28: 916-931

33 Rohleder N, Nater UM. Determinants of salivary alpha-amylase in humans and methodological considerations. Psychoneuroendocrinology 2009; 34: 469-485

34 Rohleder N, Wolf JM, Maldonado EF, Kirschbaum C. The psychosocial stress-induced increase in salivary alpha-amylase is independent of saliva flow rate. Psychophysiology 2006; 43: 645-652

35 Saruta J, Tsukinoki K, Sasaguri K, Ishii H, Yasuda M, Osamura YR, Watanabe $Y$, Sato $S$. Expression and localization of chromogranin A gene and protein in human submandibular gland. Cells Tissues Organs (Print) 2005; 180: 237-244

36 Ship JA, Fischer DJ. Metabolic indicators of hydration status in the prediction of parotid salivary-gland function. Arch Oral Biol 1999; 44: 343-350

37 van Stegeren A, Rohleder N, Everaerd W, Wolf OT. Salivary alpha amylase as marker for adrenergic activity during stress: effect of betablockade. Psychoneuroendocrinology 2006; 31: 137-141

38 Strahler J, Berndt C, Kirschbaum C, Rohleder N. Aging diurnal rhythms and chronic stress: Distinct alteration of diurnal rhythmicity of salivary alpha-amylase and cortisol. Biol Psychol 2010; 84: 248-256

39 Strahler K, Ehrlenspiel F, Heene M, Brand R. Competitive anxiety and cortisol awakening response in the week leading up to a competition. Psychol Sport Exerc 2010; 11: 148-154

40 Strub JM, Goumon Y, Lugardon K, Capon C, Lopez M, Moniatte M, Van Dorsselaer A, Aunis D, Metz-Boutigue MH. Antibacterial activity of glycosylated and phosphorylated chromogranin A-derived peptide 173194 from bovine adrenal medullary chromaffin granules. J Biol Chem 1996; 271: 28533-28540

41 Tsigos C, Chrousos GP. Hypothalamic-pituitary-adrenal axis, neuroendocrine factors and stress. J Psychosom Res 2002; 53: 865-871

42 Uesato M, Nabeya Y, Akai T, Inoue M, Watanabe Y, Kawahira H, Mamiya T, Ohta Y, Motojima R, Kagaya A, Muto Y, Hayashi H, Matsubara H. Salivary amylase activity is useful for assessing perioperative stress in response to pain in patients undergoing endoscopic submucosal dissection of gastric tumors under deep sedation. Gastric Cancer 2010; 13: $84-89$

43 Walsh NP, Montague JC, Callow N, Rowlands AV. Saliva flow rate, total protein concentration and osmolality as potential markers of whole body hydration status during progressive acute dehydration in humans. Arch Oral Biol 2004; 49: 149-154

44 Watson D, Clark LA. The PANAS-X: Manual for the positive and negative affect schedule - expanded form. Unpublished manuscript 1994; University of Iowa, Iowa City, IA.

45 Zhang K, Rao F, Wen G, Salem RM, Vaingankar S, Mahata M, Mahapatra NR, Lillie EO, Cadman PE, Friese RS, Hamilton BA, Hook VY, Mahata $S K$, Taupenot L, O'Connor DT. Catecholamine storage vesicles and the metabolic syndrome: The role of the chromogranin A fragment pancreastatin. Diabetes Obes Metab 2006; 8: 621-633 